\title{
NETWORK MANAGEMENT USING SNMP
}

\author{
Namrata \\ Department of ECE \\ R V College of Engineering Bengaluru-560059, India \\ Dr. S Ravi Shankar \\ Department of ECE \\ R V College of Engineering Bengaluru-560059, India \\ Suresh Babu Kandukuri \\ Energy management Unit \\ Siemens Technology and Services Bengaluru-560100, India
}

\begin{abstract}
Communication is one the important aspects in human life since thousands of years ago; basically, it is the transfer of information or message between two people, places or machines. Information can also be shared among different computers, routers, switches and between networks. Group of interconnected devices is called Network. Devices include computers, routers, switches, servers etc., thus network allows the sharing of data. In order to have the smooth functionality of network, it has be continuously monitored. So, network monitoring is the major role of network, in which it tries to discern low speed or unsuccessful network devices, such as failing routers, servers, switches and other malfunctioning devices. Network monitoring system cautions the occurrence of a network failure to the Network Administrator (NA). Network monitoring is predominantly put through software tools and applications. The constant and secure supervise of a network includes trace and logs of network parameters. The network parameters include data transmission rate, user-time percentages, downtime of the device, uptime of the device, response time to user and error rates.
\end{abstract}

This paper describes the network monitoring using SNMP (Simple Network Management Protocol), obtaining required system parameters like RAM usage, CPU usage and Disk usage from device which is to be monitored, storing the system values in database and displaying data using Grafana tool.

Keywords: SNMP (Simple Network Management Protocol), NA (Network Administrator), Network monitoring, System parameters, Database, Grafana tool.

Cite this Article: Namrata, S Ravi Shankar and Suresh Babu Kandukuri, Network Management Using Snmp, International Journal of Advanced Research in Engineering and Technology, 10(3), 2019, pp 81-86.

http://iaeme.com/Home/issue/IJARET?Volume $=10 \&$ Issue $=3$ 


\section{INTRODUCION}

Communication is one the important aspects in human life since thousands of years ago; basically, it is the transfer of information or message between two people, places or machines. The person who generates message is known as the source of message or sender, the generated message has to be delivered to the person at the other end who is known as the receiver. Information can also be shared among different computers, routers, switches and between networks. Group of interconnected devices is called Network. Devices include computers, routers, switches, servers etc., thus network allows the sharing of data. Internet is the best realtime example for a network.

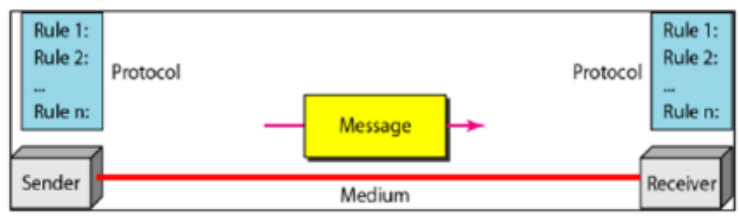

Figure 1: Basic Communication system

Network is an interconnection of many devices. Network with a smaller number of devices in it can be configured manually. Configuring of devices in the network becomes complex if the network consists of many devices interconnected to it. In order to have the smooth functionality of network, it has be continuously monitored. So, network monitoring is the major role of network, in which it tries to discern low speed or unsuccessful network devices, such as failing routers, servers, switches and other malfunctioning devices. Network monitoring system cautions the occurrence of a network failure to the Network Administrator (NA). Network monitoring is considered as the part of network management.

Network monitoring is predominantly put through software tools and applications. The constant and secure supervise of a network includes trace and logs of network parameters. The network parameters include data transmission rate, user-time percentages, downtime of the device, uptime of the device, response time to user and error rates. Alert messages are stimulated, and network fault management processes are initiated when predetermined parameter thresholds are reached. Monitoring of any network can use software based system or hardware based system or the combination of both software and hardware based system for constant monitoring. Details that are constantly observed during the monitoring of network are as follows:

- System performance

- Services

- Resources

- System changes

- System configuration

All the above parameters are tracked constantly to know the System faults and statistics.

The main concern of any IT industry in today's world is network monitoring. Network monitoring is considered as a critical IT process because all components in the network has to be constantly checked for errors and has to be evaluated continuously for smooth operation. Obtaining performance issues helps in identifying issues at the initial stage.

This paper describes about the network management and monitoring using SNMP, values of the system parameters are obtained using SNMP message commands. System values are pushed to database and values are displayed using Grafana tool. 


\section{SIMPLE NETWORK MANAGEMENT PROTOCOL}

SNMP (Simple Network Management Protocol) is an Application Layer protocol [1] [2]. We can use SNMP to get information about devices in the network like servers, computers, routers, printers, switches and services. One can obtain the information like RAM usage, CPU usage, Disk usage, System up time etc. The three main parts of SNMP communication are listed below:

- SNMP Manager

- SNMP agent

- Managed device

\subsection{SNMP Manger}

Typically this SNMP manager is the computer that remotely monitors the devices in the network by sending the request to SNMP agent. It communicates with the devices in the network through SNMP agent. It receives the responses from SNMP agent.

\subsection{SNMP Agent}

The SNMP agent is the software that is present in the network device by default. In order to monitor the device using SNMP, then the SNMP agent has to be enabled in the device. Once the agent is activated than requests from the SNMP manager can be relayed to the device through the SNMP agent and also the responses are sent back to manager from device through agent.

Every SNMP agent will have Management Information Database (MIB) that describes the parameters of the device to be monitored. This information is used by the SNMP manager to get information about particular system parameter by sending request to the SNMP agent. Thus SNMP manager should be aware of this MIB. The MIB is shared between manager and agent.

\subsection{Structure of MIB}

Agent in a network managed by SNMP should maintain the database that includes information about network. The database is known as Management Information Base. The template is used to format your paper and style the text.

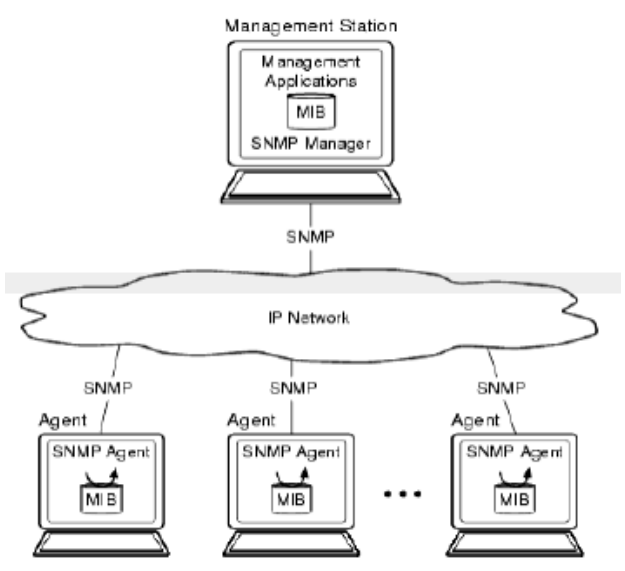

Figure 2: SNMP network [3]

From the above figure number 2, The SNMP manager and SNMP agent share the same MIB [3]. SNMP agent is enabled in the device that has to be monitored. Information about the parameters of the device in which SNMP agent is enabled is present in MIB. SNMP manager sends request to agent i.e. it requests for objects. Object is used to collect information about device in the work. There are many objects and each objects are unique. These objects are 
identified by Object Identifiers (OID). OID is a long sequence of numbers and these numbers are separated by dots. Collection of objects is called as MIB [5]. The example for MIB structure is shown below:

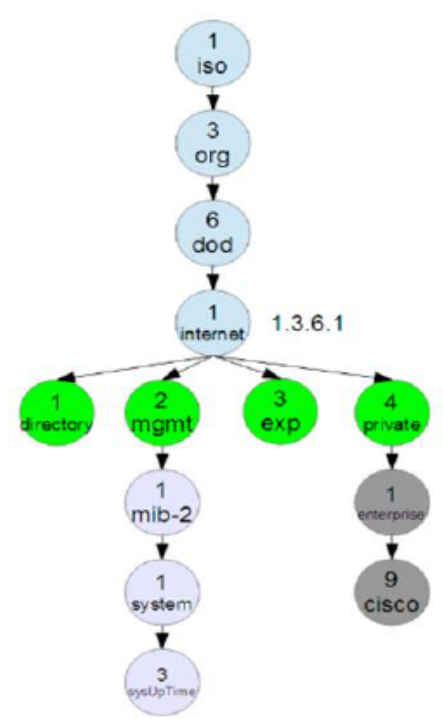

Figure 3: Structure of MIB [4]

The information system in SNMP is in the form of tree. From above figure number 3, there are many nodes. Each node has information about the object and each object has unique OID. For example the unique OID for the object sysUpTime is .1.3.6.1.2.1.1.3.O. Looking at the figure above, you can easily deduce this OID. The ' $O$ ' at the last of OID signifies that this object is a scalar and not a table[4].

There is also a textual description of the numeric OID. For example, the textual description of sysUpTime OID (presented above) is iso.org.dod.internet.mgmt.mib-2.system.sysUpTime.

\subsection{SNMP Messages}

The communication between the SNMP manger and SNMP agent takes place through following SNMP message commands:

- SNMP GET

- SNMP GET-NEXT

- SNMP GET-RESPONSE

- SNMP SET

- SNMP TRAP

This SNMP GET message is used to get the information from the network device, is sent through Protocol Data Unit (PDU). This PDU consists of OID of the particular object that SNMP manager wants to query, the response given by the object is forwarded to SNMP manger by the SNMP agent thus SNMP agent acts as the interface between the SNMP manger and the device [4].

\section{IMPLEMENTATION}

The steps for network monitoring are listed below:

Enable SNMP in device

- Pass OIDs to the device from manager by agent 
- Monitoring the device

- Get the values

- Install database

- Store the values in Database

- Install Grafana

- Make connection between database and Grafana

- Write queries to display data in the dashboard using Grafana.

Accessing the database using Grafana is shown if fig below

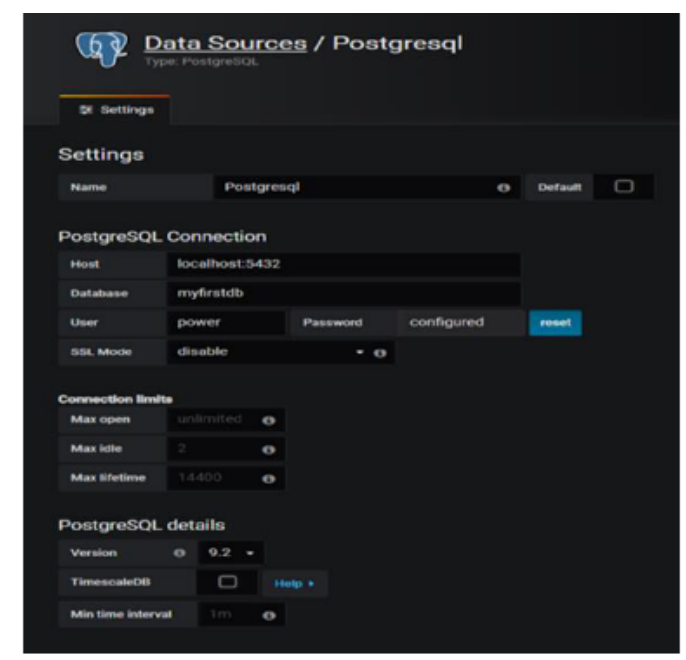

Figure 4: Database connection to Grafana

The system values stored in database are displayed in dashboard using Grafana. The dashboard created using Grafana is shown in below picture:

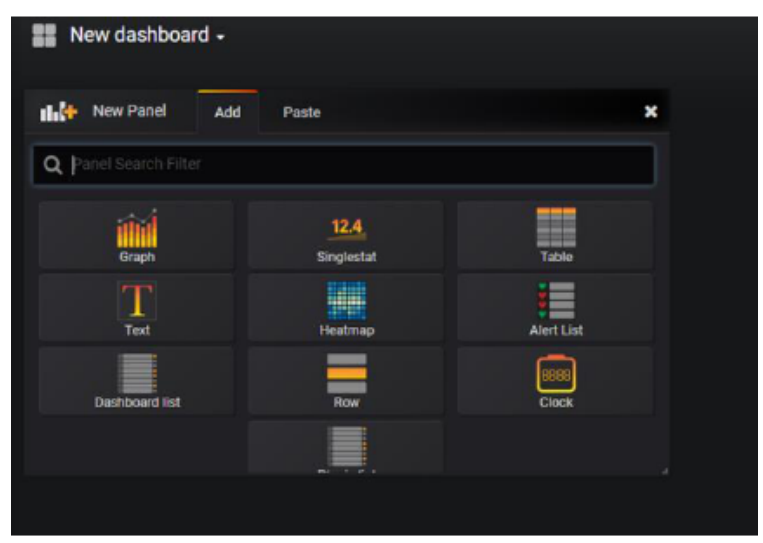

Figure 5: New dashboard using Grafana

Write queries to display data in the dashboard using Grafana.

\section{RESULTS AND DISCUSSION}

After enabling the SNMP agent in the device, the values obtained from the device is stored in database, those values are queried for displaying the data in dashboard that is created using Grafana tool. The dashboard shows the RAM usage, CPU usage, Disk usage in graphical form. The picture of final dashboard shown below: 


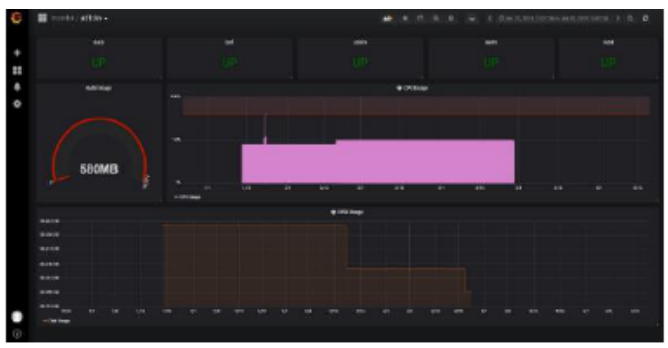

Figure 6: Dashboard of monitoring

From above figure number 6, the RAM usage, CPU usage and Disk usage are continuously monitored. The above picture has three different and unique sections to display the three parameters. Thus making user friendly GUI.

\section{CONCLUSION AND FUTURE SCOPE}

This paper gives the method for monitoring the network using SNMP. Different system parameter values are stored in database and are queried for displaying the data in dashboard that is created using Grafana tool. Obtained and displayed the values of RAM usage, CPU usage, Disk usage system parameters. In future these system values can be pushed to cloud and one can go for cloud-based UI for displaying system values

\section{REFERENCES}

[1] J.D.Case, J.R.Davin,M.S.Fedor and M.L.Schoffstall, "Internet network management using the simple network management protocol," 14th Conference on Local Computer Networks 1989.

[2] A. Ben-Artzi, A. Chandna and U. Warrier, "Network management of TCP/IP networks: present and future", IEEE Network, Volume: 4, Issue: 4, 1990.

[3] SNMPMIB,“Oracle”[Online].Available:https://docs.oracle.com/cd/E13203_01/tuxedo/tux81/s nmpmref/1 tmib.htm

[4] SNMPtutorial,"ManageEngine”[Online].Avilable:https://www.manageengine.com/networkmonitoring/what-is-snmp.html

[5] Katker, Paterok, Vogt, Wittig and Delgrossi,R. Nicole, "An SNMP MIB for the ST-II Protocol", Proceedings of IEEE International Conference on Multimedia Computing and Systems. 1994

[6] Sarvesh Kumar Tripathi, Dr Avjeet Kaur, Dr Rk Pandey And Dr Meghna Chhabra Time To Modify Operating Software (Os), Databases (Db) And Tcp/Ip Protocols for Data Trash Elimination, Based on User Defined Shelf Life of Data. International Journal of Computer Engineering and Technology, 10(1), 2019, pp. 138-156

[7] Manjunath Kotari, Sunil Manohar Dasharathi and Dr. Niranjan N. Chiplunkar, Implementation of Customized Network Monitoring Tool and Security Framework Monitoring System, International Journal of Computer Engineering \& Technology (IJCET), Volume 5, Issue 9, September (2014), pp. 37-49

[8] P. Naveen Kalyan and K. Jaya Swaroop, Amba-Axi Protocol Verification by Using UVM, International Journal of Electronics and Communication Engineering and Technology, 7(4), 2016, pp. 76-84

[9] Mostafa M. El-Said, An Empirical Study to Compare Between IEEE 802.11p And Wave Protocols in Vanets Networks, International Journal of Computer Engineering and Technology (IJCET), Volume 4, Issue 4, July-August (2013), pp. 547-555

[10] Anjumol O N and P.K.K Thampi, An Energy Efficient Protocol Based Study of WSN to Increase the Lifetime, International Journal of Computer Engineering and Technology (IJCET), Volume 5, Issue 12, December (2014), pp. 70-75

[11] M.Venkatesan and Dr.S.Selvakumar Raja, An Implementation of Invanet Hybrid Architecture and Hybrid Routing Protocol Using Deep Neural Network, International Journal of Mechanical Engineering and 2018, pp. 377-386 